\title{
Does the length of specimen storage affect influenza testing results by real-time reverse transcription- polymerase chain reaction? An analysis of influenza surveillance specimens, 2008 to 2010
}

D L Caselton (dcaselton@gmail.com) ${ }^{1}$, G Arunga ${ }^{1}$, G Emukule ${ }^{1}$, P Muthoka² ${ }^{2}$ L Mayieka ${ }^{1}$, A Kosgey ${ }^{1}$, R Ochola $^{1}$, L W Waiboci $^{1}$,

D R Feikin'3, J A Mott', R F Breiman', M A Katz ${ }^{1}$

1. Influenza Program, Global Disease Detection Division, Centers for Disease Control and Prevention, Kenya

2. Kenya Ministry of Public Health and Sanitation, Kenya

3. International Emerging Infections Program, Global Disease Detection Division, Centers for Disease Control and Prevention, Kenya

Citation style for this article:

Caselton DL, Arunga G, Emukule G, Muthoka P, Mayieka L, Kosgey A, Ochola R, Waiboci LW, Feikin DR, Mott JA, Breiman RF, Katz MA. Does the length of specimen storage affect influenza testing results by real-time reverse transcription-polymerase chain reaction? An analysis of influenza surveillance specimens, 2008 to 2010. Euro Surveill. 2014;19(36):pii=20893. Available online: http://www.eurosurveillance.org/ViewArticle.aspx?Articleld=20893

In some influenza surveillance systems, timely transport to laboratories for reverse transcription-polymerase chain reaction (RT-PCR) testing is challenging. Guidelines suggest that samples can be stored at $4^{\circ} \mathrm{C}$ for up to 96 hours but the effect of longer storage times has not been systematically evaluated. We collected nasopharyngeal and oropharyngeal specimens from patients in Kenya and stored them in viral transport medium at 2 to $8^{\circ} \mathrm{C}$ before testing for influenza $A$ and $B$ using real-time RT-PCR. From April 2008 to November 2010, we collected 7,833 samples; 940 (12\%) were positive for influenza. In multivariable analysis, specimens stored for six days were less likely to be influenza-positive compared to specimens stored between zero and one day (adjusted odds ratio (aOR): 0.49, 95\% confidence interval ( $\mathrm{Cl}): 0.27-0.93)$. There was no statistically significant difference in influenza positivity of specimens stored for five days compared to zero to one day. There was no statistically significant relationship between days in refrigeration and cycle threshold $\left(C_{t}\right)$ values for positive samples $(p=0.31)$. We found that samples could remain in storage for at least five days without affecting the proportion-positive of samples, potentially increasing the feasibility of including influenza surveillance sites in remote areas.

\section{Introduction}

Worldwide, an estimated 28,000 to 111,500 deaths attributable to influenza-associated acute lower respiratory infections occurred in children under five yearsold in 2008 [1]. In recent years, with increased concerns about detecting and responding to an influenza pandemic, influenza surveillance has expanded globally. Many surveillance sites in resource-poor countries are remote and lack onsite diagnostic capacity, requiring that samples be transported far distances to a central laboratory $[2,3]$. In influenza surveillance systems throughout the world, real-time reverse transcriptionpolymerase chain reaction (rRT-PCR) is increasingly used as the test of choice to confirm influenza virus infection [4]. However, little is known about the optimal time that specimens can be stored in a refrigerator before being tested by rRT-PCR and recommendations regarding the maximum length of storage time of respiratory specimens before such assays vary among institutions. The World Health Organization (WHO) and the United States (US) Centers for Disease Control and Prevention $(C D C)$ recommend that a sample be stored for no more than four days at $4^{\circ} \mathrm{C}$ before freezing or diagnostic testing $[5,6]$ but neither guideline cites evidence as the basis for this recommendation, and a literature search revealed no published manuscripts on this topic.

In Kenya, as in many other countries in Africa, surveillance for influenza is conducted in healthcare facilities that are far from the laboratory that processes the specimens, and timely transport of specimens is often challenging. As a result, specimens sometimes may be stored at $4^{\circ} \mathrm{C}$ for longer than 96 hours. In order to address the question of whether prolonged refrigeration might lead to virus deterioration, which in turn would be associated with lower rates of positivity and lower overall viral loads, we evaluated two-anda-half years of influenza surveillance data from Kenya to determine the relationship between the number of days a specimen was kept in storage and detection of influenza positivity by molecular testing. In addition, we evaluated the relationship between the number of storage days and the cycle threshold $\left(C_{t}\right)$ values of influenza-positive samples. 


\section{Methods}

\section{Data collection}

Influenza sentinel surveillance system

In 2007, the Kenya Ministry of Public Health and Sanitation (MoPHS) and the Kenya Medical Research Institute/Centers for Disease Control and PreventionKenya (KEMRI/CDC) established a national influenza sentinel surveillance system in Kenya in order to better understand the seasonality, burden, and epidemiology of influenza in the country and to detect new influenza virus strains with pandemic potential and for possible use in new vaccine formulations. At each of the sentinel healthcare facilities, a trained surveillance officer collects nasopharyngeal (NP) and oropharyngeal (OP) samples from all hospitalised patients with severe acute respiratory illness (SARI) and from up to three outpatients a day with influenza-like illness (ILI). For this study verbal consent was obtained from all patients before questionnaires were administered and specimens were collected. For children, verbal consent was obtained from guardians. Case definitions for SARI and ILI and the procedure for specimen collection have been previously described [7].

Specimen storage and transport

NP and OP specimens from each patient were placed in the same cryovial with viral transport medium (VTM). VTM was prepared centrally at the KEMRI/CDC laboratory using a WHO protocol that includes bovine serum albumin and veal infusion broth supplemented with amphotericin B [8]. Briefly, $10 \mathrm{~g}$ veal infusion broth, $2 \mathrm{~g}$ of bovine albumin fraction $\mathrm{V}$ and $3.2 \mathrm{ml}$ of fungizone $(250 \mu \mathrm{g} / \mathrm{ml}$ amphotericin) were weighed and 400 $\mathrm{ml}$ of distilled water added and the contents allowed to dissolve by gentle stirring. The media was allowed to stand for one hour at $4^{\circ} \mathrm{C}$, sterilised by filtration, and using aseptic techniques, $1 \mathrm{ml}$ aliquots dispensed into sterile $1.8 \mathrm{ml}$ propylene cryovials. Quality control steps were included at all steps in the VTM preparation. The VTM was shipped, at 2 to $8^{\circ} \mathrm{C}$, to surveillance sites and refrigerated prior to and after insertion of the swab specimens. VTM was used for up to three months after preparation. After collection, specimens in VTM were immediately placed in refrigeration at 2 to $8^{\circ} \mathrm{C}$. All specimens were transported in cool boxes by road to the National Influenza Center (NIC) in Nairobi and were tested for influenza by rRT-PCR at KEMRI/CDC laboratory in Nairobi. The cool boxes were kept at refrigeration temperature with ice packs. Upon arrival at the laboratory all specimens were frozen at $-80^{\circ} \mathrm{C}$.

\section{Laboratory testing}

An aliquot of each respiratory specimen was tested by $r R T-P C R$ for influenza $A$ and influenza $B$ after one freeze-thaw cycle. Specimens positive for influenza $A$ were subtyped for seasonal $\mathrm{H}_{1}$ and $\mathrm{H}_{3}$ as well as for $\mathrm{H}_{5}$ and $\mathrm{A}\left(\mathrm{H}_{1} \mathrm{~N}_{1}\right)$ pdmog by rRT-PCR [6]. Samples were aliquoted and total RNA was extracted from $100 \mu \mathrm{l}$ aliquots of each sample using QIAamp viral RNA minikit
Sentinel influenza surveillance sites in Kenya, 2008-2010 $(n=7)$

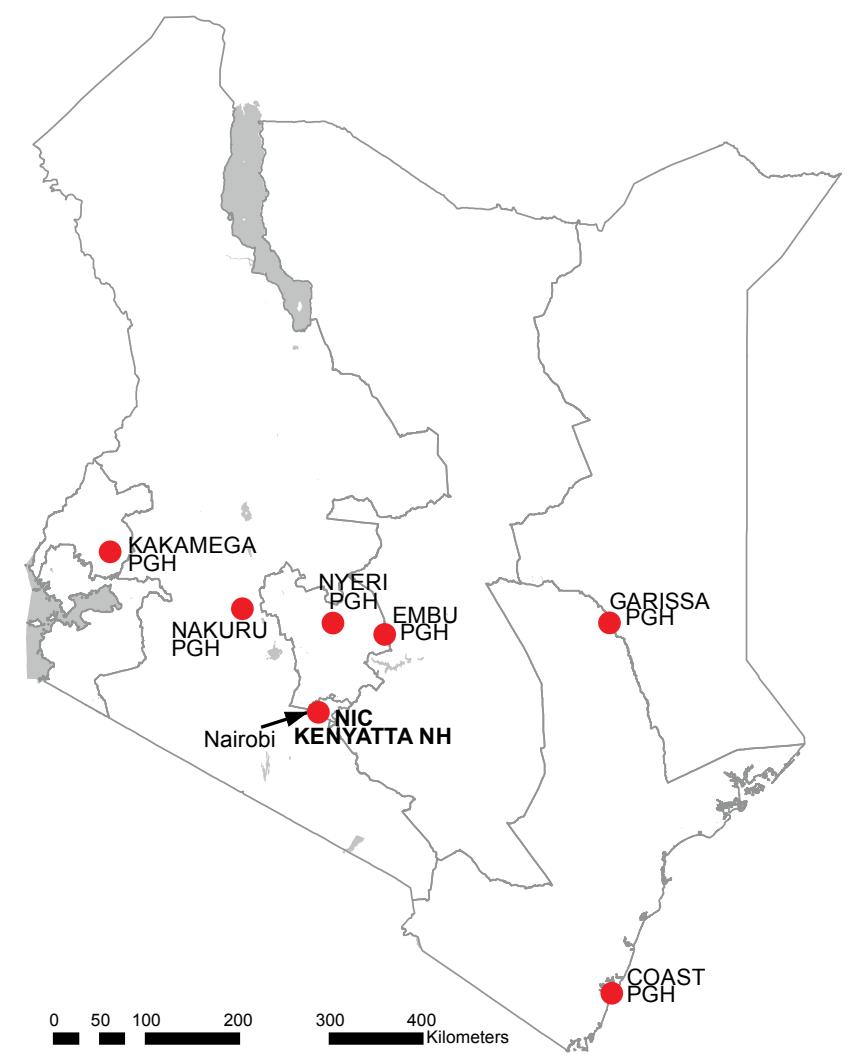

NH: national hospital; NIC: National Influenza Center; PGH: provincial general hospital.

(Qiagen inc, Valencia CA, USA), according to manufacturer's instructions. One step rRT-PCR was carried out using AgPath kits (Applied Biosystems, California USA). The primers, probes, and positive controls for all influenza viruses were provided by CDC-Atlanta [9]. Following the reverse transcription step, a typical 45 cycle PCR reaction was run and fluorescence was read at the annealing/extension step at $55^{\circ} \mathrm{C}$, and recorded at each cycle [10]. Appropriate negative and positive control specimens were run alongside each reaction. The results were recorded as cross-over $C_{t}$ values. $A C_{t}$ value $\leq 39.9$ was regarded as positive, whereas $C_{t}$ values $\geq 40.0$ were considered negative in the analysis. Samples with no $C_{t}$ values were regarded as negative [9].

\section{Data analysis and statistical methods}

Samples

Samples collected from seven sentinel surveillance sites between 10 April 2008 and 8 November 2010 with available storage, demographic, and laboratory data were included in the analysis. The seven sites were located from $2 \mathrm{~km}$ to $487 \mathrm{~km}$ from the laboratory in Nairobi (Figure 1). We determined the number of storage days that samples were refrigerated by subtracting 
the date the sample was collected from the date the sample was received at the NIC laboratory. Transport time was included in the refrigeration period. Samples that were in refrigeration for duration of zero to 10 days were included in the analysis.

\section{Statistical analysis}

Storage days were analysed as individual days. Because a relatively small number of samples were stored for only one day and there was no difference in influenza positivity between zero and one storage days (p>0.05), we combined zero and one storage days into one category to use as a reference group. We used the Cochran-Armitage trend test to assess the relationship between storage days and positivity of influenza samples. We then modeled influenza positivity with storage day as a categorical predictor (0-1 vs 2, 3, 4, 5, 6, $7,8,9,10)$ using multivariable logistic regression, controlling for the following variables: patient age, days since illness onset, surveillance site, and syndrome (ILI or SARI) classification. If the variable was found to be associated with influenza positivity at p<0.2 in the bivariate analysis it was considered a potential confounder and included in the multivariable analysis. We included days since illness onset in the model because the quantity of viral shedding decreases after three to five days following illness onset [11]. We then fit the logistic regression model for ILI and SARI cases (controlling for patient age, days since illness onset, surveillance site). We used logistic regression rather than linear regression because we felt that logistic regression was the best way to assess the effect of the length of specimen storage on influenza positivity; in our analysis, using logistic regression allowed us to target the outcome variable (influenza positivity), which was dichotomous and categorical.

In order to determine the relationship between storage days and $C_{t}$ values of influenza samples we performed multivariable generalised ordinal logit analysis for influenza-positive samples. We excluded the 39 specimens that had influenza $A / B$ co-infections because including two $C_{t}$ values for an individual sample would have made it impossible to draw a single conclusion about the relationship between storage time and $C_{t}$ value for the sample. $C_{t}$ values were not normally distributed; therefore we created quartiles for $C_{t}$ values, and a multivariable generalised ordinal logit model was fit using zero to one as a reference group. We also analysed the mean $C_{t}$ values for influenza-positive samples by storage day. We stratified the positive results by $C_{t}$ values using the categories $\leq 29,30-\leq 37$, and 38-<40 [12]. Data analysis was done using SAS version 9.1 (SAS Institute Inc, Cary, North Carolina, USA) and findings were considered statistically significant if the $p$-value was $<0.05$.

Ethical considerations

The Kenyan Ministry of Health determined that the surveillance system was part of routine national disease surveillance and did not represent research and did not require ethical review.

\section{Results}

\section{Demographics}

Of 12,541 samples collected during the study period in the seven sites, 7,833 (62\%) had storage, demographic, and testing data available and were included in the analysis. Of the 7,833 samples, 940 (12\%) were positive for influenza; 718 ( $9 \%)$ were influenza A only, $183(2 \%)$ were influenza B only, and 39 ( $\$ 1 \%)$ were positive for both $A$ and $B$. Of the 528 influenza A-positive samples that were subtyped, $95(18 \%)$ were seasonal $\mathrm{H}_{1}, 222$ (42\%) were seasonal $\mathrm{H}_{3}$, and 211 (40\%) were $\mathrm{A}\left(\mathrm{H}_{1} \mathrm{~N}_{1}\right)$ pdmo9; 4,311 samples (55\%) were from male patients. The mean age was 2.4 years, and the majority of samples $(5,095 ; 65 \%)$ were from patients $<2$ years-old. Of the 4,708 samples that were not included because storage and/or testing data were not available, 3,568 (76\%) had age data and, 3,624 (77\%) had

\section{TABLE 1}

Demographic characteristics and influenza positivity among influenza-like illness and severe acute respiratory illness patients tested for influenza infections, Kenya, 2008-2010 $(\mathrm{n}=7,833)$

\begin{tabular}{|c|c|c|}
\hline Variable & Number tested (\%) & $\begin{array}{c}\text { Number positive for } \\
\text { Influenza (\%) }\end{array}$ \\
\hline \multicolumn{3}{|c|}{ Age group in years } \\
\hline$<2$ & $5,095(65)$ & $493(10)$ \\
\hline $2-4$ & $2,147(27)$ & $331(15)$ \\
\hline $5-17$ & $454(6)$ & $102(22)$ \\
\hline$\geq 18$ & $137(2)$ & $14(10)$ \\
\hline Total & $7,833(100)$ & $940(12)$ \\
\hline \multicolumn{3}{|l|}{ Sex } \\
\hline Male & $4,311(55)$ & $511(12)$ \\
\hline Female & $3,522(45)$ & $429(12)$ \\
\hline Total & $7,833(100)$ & $940(12)$ \\
\hline \multicolumn{3}{|c|}{ Sentinel site } \\
\hline Embu & $474(6)$ & $41(9)$ \\
\hline Garissa & $383(5)$ & 49 (13) \\
\hline Kakamega & $2,383(30)$ & $264(11)$ \\
\hline Kenyatta & $478(6)$ & $33(7)$ \\
\hline Coast & $840(11)$ & $73(9)$ \\
\hline Nakuru & $1,433(18)$ & $196(14)$ \\
\hline Nyeri & $1,842(24)$ & $281(15)$ \\
\hline Total & $7,833(100)$ & $940(12)$ \\
\hline \multicolumn{3}{|l|}{ Case type } \\
\hline $\mathrm{ILI}$ & $3,813(48)$ & $561(15)$ \\
\hline SARI & $4,012(51)$ & 378 (9) \\
\hline Total & $7,825^{a}(100)$ & $939^{a}(12)$ \\
\hline
\end{tabular}

ILI: influenza-like illness; SARI: severe acute respiratory illness.

Eight samples were missing data on SARI and ILI categorisation. One of the samples with missing data was positive for influenza. 


\section{TABLE 2}

Association between duration of storage of respiratory samples and percentage of influenza A and B-positive using zero to one storage days as a reference, Kenya, $2008-2010$

\begin{tabular}{|c|c|c|c|}
\hline \multirow{2}{*}{$\begin{array}{l}\text { Storage } \\
\text { time in } \\
\text { days }\end{array}$} & \multirow{2}{*}{$\begin{array}{c}\mathrm{n} / \mathrm{N}(\% \\
\text { positive) }\end{array}$} & Bivariate analysis & $\begin{array}{c}\text { Multivariable } \\
\text { analysis }^{a}\end{array}$ \\
\hline & & OR $(95 \% \mathrm{Cl})$ & aOR $(95 \% \mathrm{Cl})$ \\
\hline $0-1$ & $462 / 3,969$ (12) & REF & REF \\
\hline 2 & $240 / 1,899$ (13) & $1.10(0.93-1.30)$ & $0.98(0.83-1.17)$ \\
\hline 3 & 79/617 (13) & $1.12(0.86-1.44)$ & $0.96(0.74-1.25)$ \\
\hline 4 & $103 / 673(15)$ & $1.37(1.09-1.73)^{\mathrm{b}}$ & $1.26(1.00-1.61)$ \\
\hline 5 & $26 / 222(12)$ & $1.01(0.66-1.53)$ & $0.98(0.66-1.54)$ \\
\hline 6 & $11 / 180(6)$ & $0.49(0.27-0.92)^{b}$ & $0.49(0.27-0.93)^{b}$ \\
\hline 7 & $8 / 119(7)$ & $0.55(0.27-1.13)$ & $0.50(0.25-1.07)$ \\
\hline 8 & $7 / 67$ (10) & $0.89(0.40-1.95)$ & $0.88(0.40-1.94)$ \\
\hline 9 & $2 / 47(4)$ & $0.34(0.08-1.40)$ & $0.33(0.08-1.35)$ \\
\hline 10 & $2 / 40(5)$ & $0.4(0.10-1.66)$ & $0.36(0.09-1.53)$ \\
\hline
\end{tabular}

aOR: adjusted odds ratio; $\mathrm{Cl}$ : confidence interval; OR: odds ratio; REF: reference category.

Logistic regression model controlling for patient age, days since illness onset, surveillance site, and syndrome classification (influenza-like illness vs severe acute respiratory illness); 7,792 samples were used In the multivariate analysis; eight samples had missing syndrome classification and 33 had missing data for days since illness onset.

b Statistically significant.

data on sex. For these samples, the mean age was 2.8 years and 2,036 (56\%) were from males. There was no significant difference in patient age and sex distribution between the analysed and non-analysed samples. Of the 7,833 tested samples, the majority were received from the following sentinel sites: Kakamega $(n=2,383$; $30 \%)$, Nyeri $(n=1,842 ; 24 \%)$, and Nakuru $(n=1,433$; $18 \%)$. Nearly half $(n=3,813 ; 49 \%)$ of the 7,825 samples that had clinical data were from ILI cases (Table 1).

\section{Influenza positivity and storage time}

Overall, $3,969(51 \%)$ of specimens included in the analysis were stored for zero to one day; 3,411 (44\%) of specimens analysed were stored for two to five days; and $453(6 \%)$ were stored for six to 10 days.

In the bivariate analysis, the per cent positivity of samples stored for zero to one day (12\%) was not significantly different from that of two, three, four, and five days ( $13 \%, 13 \%, 15 \%$, and $12 \%$, Table 2$)$. In the bivariate analysis, there was a statistically significant difference in the per cent-positivity of samples stored for zero to one day compared with samples stored for six days $(12 \%$ vs $6 \%, p=0.03)$. The per cent positive of samples stored for zero to one day (12\%) was higher than the per cent positive of samples stored for seven, eight, nine, and 10 days $(7 \%, 10 \%, 4 \%$, and $5 \%$, respectively) but these differences were not statistically significant (Table 2). Overall, the Cochran-Armitage trend test showed that the positivity of influenza decreased as the storage days increased ( $p<0.05)$.

In the multivariable model, the positivity of samples stored for five days did not differ from that of zero to one day ( $12 \%$ vs $12 \%$; adjusted odds ratio (aOR): $0.98 ; 95 \%$ confidence interval (CI): 0.66-1.54). Samples stored for six days were significantly less likely to be positive compared with samples stored for zero to one day ( $6 \%$ vs $12 \%$; aOR: 0.49; $95 \% \mathrm{Cl}$ : 0.27-0.93). Samples stored for seven days were less likely to be positive as well, but this finding did not reach statistical significance ( $7 \%$ vs $12 \%$; aOR: 0.50 ; $95 \% \mathrm{Cl}$ : $0.25-1.07$ ). Samples stored for eight, nine, and 10 days respectively were less likely to be positive than those samples stored for zero to one day, but these findings were not statistically significant and had wide confidence intervals due to the small sample size (Table 2). We compared specimens stored for zero to one day with specimens stored for eight to 10 days using a multivariable model, and samples stored for eight to 10 days were less likely to be positive for influenza than those in storage for zero to one day ( $7 \%$ vs $12 \%$; aOR:0.56; $95 \% \mathrm{Cl}$ : $0.03-$ 1.05). Additionally, in the multivariable model, samples stored for six to 10 days were $49 \%$ less likely to be positive than those stored for zero to five days ( $7 \%$ vs $12 \%$; aOR: 0.51; 95\% Cl: 0.35-0.75).

In a multivariable analysis comparing storage time and positivity rates of influenza $A$, we found no statistically significant difference in the positivity rates of specimens stored for two, three, four and five days compared to specimens stored for zero to one day. However, specimens stored for six days were less likely to be positive for influenza A compared to specimens stored for zero to one day $(8 / 180$ (4\%) vs $353 / 3,969$ (9\%); aOR: $0.48 ; 95 \% \mathrm{Cl}: 0.23-0.99)$. There was no difference in the positivity rates between specimens stored for seven, eight, nine and 10 days and those stored for zero to one day, but only 17 specimens stored for seven to 10 days were positive for influenza $A$. The trend test showed no trend between storage days and positivity rates for influenza $A$ (p>0.05).

We conducted a multivariable analysis comparing storage time and positivity rates of the influenza $A$ subtypes, and we found similar trends in positivity rates, although the analysis was limited by the small sample size. For H1, compared to specimens stored for zero to one days, there was no statistically significant difference in the positivity rates of samples stored for three, four, five, six, and seven to 10 days, but samples stored for two days were twice as likely to be negative $(17 / 1,899(1 \%)$ vs $60 / 3,969(2 \%)$; aOR: $0.50 ; 95 \%$ $\mathrm{Cl}: 0.30-0.90)$. For $\mathrm{H}_{3}$, we found no statistically significant difference in the positivity rates of specimens stored for $>1$ day compared to specimens stored for zero to one day. Finally, for $A\left(\mathrm{H}_{1} \mathrm{~N}_{1}\right)$ pdmog, the positivity rates of specimens stored for $>1$ day were similar to the positivity rates of specimens stored for zero to one day with one exception: samples stored for four days were 
Mean cycle threshold $\left(\mathrm{C}_{\mathrm{t}}\right)$ values of influenza positive samples relative to the number of storage days, Kenya, 2008-2010 $(\mathrm{n}=901)^{\mathrm{a}}$

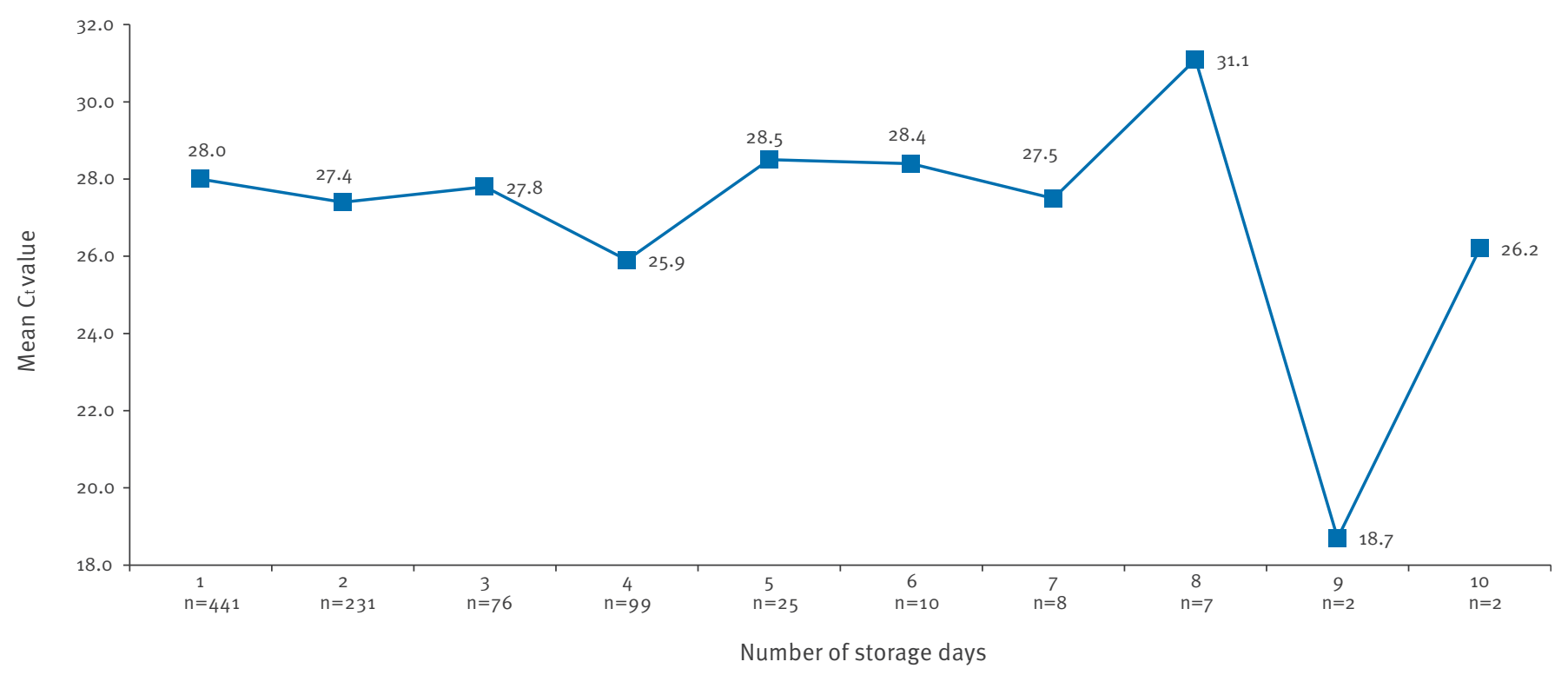

The number of samples (n) stored for the respective amount of storage days is indicated under the $\mathrm{X}$ axis values.

a Does not include 39 co-infections.

twice as likely to be positive compared to those stored at zero to one day $(33 / 673(5 \%)$ vs $86 / 3,969(2 \%)$; aOR: 2.33 ; $95 \% \mathrm{Cl}: 1.52-3.56)$. Overall, the CochranArmitage Trend test showed that the positivity of influenza decreased as the storage days increased ( $p<0.05)$ for $\mathrm{H}_{1}$ and $\mathrm{A}\left(\mathrm{H}_{1} \mathrm{~N}_{1}\right)$ pdmog, but not for $\mathrm{H}_{3}$.

In a multivariable analysis of storage time and influenza $B$, there were no statistically significant differences in positivity rates by storage time. However, numbers were small; only 12 specimens stored for five to 10 days were positive for influenza B. There was no significant trend between storage days and the positivity rates for influenza B ( $p>0.05)$. When we analysed specimens from ILI and SARI cases separately, in the multivariable analysis, there was no significant decrease in the proportion-positive of samples stored for any number of storage days compared with those stored for zero to one day.

\section{Cycle threshold value and storage time}

We analysed 901/940 (96\%) of all positive samples. We excluded 39 positive samples because they had influenza $A$ and influenza $B$ co-infections. The $C_{t}$ values ranged from 12.66 to 39.99 . In the multivariable generalised ordinal logit analysis, $C_{t}$ values from influenza-positive specimens stored for >1 day were not significantly higher than influenza-positive specimens stored for zero to one day ( $p>0.05$, Figure 2). Stratifying the positive results by $C_{t}$ values showed that $599(66 \%)$ of the 901 positive samples analysed had $C_{t}$ results of $\leq 29,178(20 \%)$ had $C_{t}$ results in the range of 30 to $\leq 37$, and $124(14 \%)$ specimens had $C_{t}$ results in the range 38 to $<40$. The distribution of the $C_{t}$ values of the positive samples was relatively consistent for each day of storage.

\section{Discussion}

While existing guidelines recommend that samples can be stored at $4^{\circ} \mathrm{C}$ for up to 96 hours before being tested influenza by rRT-PCR, our results suggest that maintaining samples at refrigeration temperature for up to five days after collection is unlikely to compromise results by rRT-PCR. The implications of our study are potentially relevant for influenza diagnostic testing throughout Kenya and other countries where surveillance systems have adopted the use of rRT-PCR as the diagnostic of choice for influenza viruses. In influenza surveillance systems that use rRT-PCR and collect samples during weekdays only, weekly transport of specimens on Friday - if samples could arrive at the laboratory the same day or the following day - would not compromise specimen integrity. This flexibility in the frequency of transport may be especially useful in rural areas, where frequent transport of samples to central laboratories can be challenging and costly.

Our analysis included nearly 8,000 specimens, and we controlled for confounding variables. The per centpositivity of samples was similar through five days. Samples that were stored for more than five days had reduced odds of testing positive for influenza relative to the reference group of zero to one day. There were few samples stored for eight to 10 days, making 
it difficult to assess these longer periods of storage. Likewise, our sub-analyses were limited by small sample size; although we found consistent positivity trends in our sub-analysis of $\mathrm{H}_{1}$ and $\mathrm{A}\left(\mathrm{H}_{1} \mathrm{~N}_{1}\right)$ pdmog, the trend was not statistically significant for $\mathrm{H}_{3}$, influenza $A$, or influenza B.

Influenza-positive specimens with higher $C_{t}$ values by rRT-PCR present more difficulty for influenza virus isolation. We have previously shown that the rates of isolation for PCR-positive samples are lower for $C_{t}$ values >35 for influenza $A$ and $C_{t}$ values >30 for influenza $B$ [13]. In our surveillance system in Kenya, culture is attempted only for samples with a $C_{t}$ value $\leq 35$. In our analysis, although the mean $C_{t}$ values of influenzapositive samples increased after four days of storage with the exception of the mean $C_{t}$ value for samples stored for nine days, there was no statistically significant association between these $C_{t}$ values and storage day. However, there were a relatively small number of influenza samples stored for five to 10 days. Because we did not evaluate isolation rates by storage time, we were unable to draw conclusions about the relationship of storage time and viral isolation. In a previous study conducted in the US that tested respiratory samples for influenza using virus isolation, there was no difference in the percentage of influenza-positive samples by storage days when samples were stored for as long as five days at $4^{\circ} \mathrm{C}[14]$. Our findings that $C_{t}$ values of influenza samples did not vary by days of storage were also similar to those of a previous study of influenza $A$ in wild birds; in that study, $C_{t}$ values of influenza-positive samples tested by rRT-PCR were similar in samples maintained at $4^{\circ} \mathrm{C}$ for up to three weeks [15].

For remote surveillance sites where specimen transport is challenging, there are storage options other than traditional VTM that could be considered, particularly when culturing specimens is not a main objective. Samples could be divided into two aliquots at the site. One aliquot could be placed in VTM and the other could be placed in lysis buffer before transport to the laboratory, which would reduce the need for prompt transport to the laboratory for PCR detection. If specimens will not ultimately be cultured, which is currently the situation in some countries in Africa, they can be stored in ethanol at room temperature without reducing the yield by PCR [16] or collected dry or placed in saline and stored in $4^{\circ} \mathrm{C}$ or ambient temperature [17]. In our surveillance system, these alternative storage methods were not a suitable option; if we had employed any of these methods we would not have been able to culture PCR-positive specimens.

The findings from this analysis are subject to limitations. First, because data associated with specimen collection and testing were incomplete, 4,708 (37\%) specimens could not be used in the analysis. However, we included nearly 8,000 samples in our evaluation, and the mean age and sex distribution were similar between those samples included and those excluded.
Second, it is possible that samples were stored and transported under conditions outside the recommended storage temperature of 2 to $8^{\circ} \mathrm{C}$. However, even if this occurred, it is unlikely that this variability affected samples stored for different periods of time differently, because samples of different collection dates were placed in the same cool box for transport. In addition, based on the schedule followed for each site, transport time from site to laboratory was consistent for every site throughout the study. In addition, we only evaluated specimen positivity by rRT-PCR, so our findings may not be applicable where other testing methods are used. However, our findings of consistent $C_{t}$ values across storage times, particularly for specimens stored for zero to five days, of which there were many, lead us to believe that isolation rates would not be affected by up to five days of storage. In addition, while our sample was large, we compared different samples rather than testing the same samples over multiple days, which would be the ideal way to evaluate variability of test results according to refrigeration time. Finally, we only tested for influenza, and therefore our results are not generalisable to other pathogens.

Our results suggest that respiratory samples can be stored at 2 to $8^{\circ} \mathrm{C}$ for up to five days after collection before reaching the laboratory; this finding could ease the burden of specimen transport in surveillance systems where sampling sites are far from the laboratory or budget for specimen transport is limited. Further studies should be conducted to better understand the association between duration of specimen storage prior to rRT-PCR testing of influenza and other viruses and bacteria as well as the effect of refrigeration storage time on virus isolation rates.

\section{Acknowledgements}

The authors wish to acknowledge the contributions of the field surveillance staff and the KEMRI/CDC laboratory personnel without whom this work would not be possible. We also thank the Kenya Ministry of Public Health and Sanitation for its collaboration on influenza sentinel surveillance. We also would like to thank John Williamson for his input in the statistical analyses, Barry Fields and Njenga Kariuki for their review of the laboratory aspects of the manuscript, and Daniel Macharia for his assistance with the surveillance map.

\section{Conflict of interest}

None declared.

Authors' contributions

D. Caselton contributed to the overall design of the analysis, analysed the data, and wrote the manuscript. G. Arunga coordinated the data entry, analysed the data, and reviewed the manuscript. G. Emulke supervised data entry and analysis. L. Waibochi, L. Mayieka, and A. Kosgey were responsible for all the PCR-testing and reviewed the manuscript. R. Ochola contributed to the data collection and reviewed the manuscript. J. Mott contributed to the study design and reviewed the manuscript. D. Feiken and R. Brieman contributed to the 
overall design of the influenza sentinel surveillance system, the design of the analysis, and reviewed the manuscript. $M$. Katz contributed to the overall design of the influenza sentinel surveillance system, the study design, the design of the analysis, and reviewed the manuscript.

\section{References}

1. Nair H, Brooks WA, Katz M, Roca A, Berkley JA, Madhi SA, et al. Global burden of respiratory infections due to seasonal influenza in young children: a systematic review and metaanalysis. Lancet. 2011;378(9807):1917-30. http://dx.doi. org/10.1016/S0140-6736(11)61051-9 http://dx.doi.org/10.1016/ So140-6736(11)61051-9

2. Mmbaga VM, Mwasekaga MJ, Mmbuji P, Matonya M, Mwafulango A, Moshi S, et al. Results from the first 30 months of national sentinel surveillance for influenza in Tanzania, 2008-2010. J Infect Dis. 2012;206 Suppl 1:S80-6. http:// dx.doi.org/10.1093/infdis/jis540

3. Nyatanyi T, Nkunda R, Rukelibuga J, Palekar R, Muhimpundu MA, Kabeja A, et al. Influenza sentinel surveillance in Rwanda, 2008-2010. J Infect Dis. 2012;206 Suppl 1:S74-9. http://dx.doi. org/10.1093/infdis/jis574

4. Lieberman D, Lieberman D, Shimoni A, Keren-Naus A, Steinberg R, Shemer-Avni Y. Identification of respiratory viruses in adults: nasopharyngeal versus oropharyngeal sampling. J Clin Microbiol. 2009;47(11):3439-43. http://dx.doi. org/10.1128/JCM.00886-09

5. Centers for Disease Control and Prevention (CDC). Interim Guidance on Specimen Collection, Processing, and Testing for Patients with Suspected Novel Influenza A ( $\left.\mathrm{H}_{1} \mathrm{~N}_{1}\right)$ Virus Infection 2009. Atlanta: CDC. [Accessed 6 July 2011]. Available from: http://www.cdc.gov/h1n1flu/specimencollection.htm

6. WHO Collaborating Centre for influenza at CDC. CDC protocol of realtime RTPCR for influenza $A\left(\mathrm{H}_{1} \mathrm{~N}_{1}\right)$ Geneva: World Health Organization; 2009. [Accessed 6 July 2011]. Available from: http://www.who.int/csr/resources/publications/swineflu/ CDCRealtimeRTPCR_SwineH1Assay-2009_20090430.pdf

7. Kim C, Ahmed JA, Eidex RB, Nyoka R, Waiboci LW, Erdman D, et al. Comparison of Nasopharyngeal and Oropharyngeal Swabs for the Diagnosis of Eight Respiratory Viruses by Real-Time Reverse Transcription-PCR Assays. PLoS One. 2011;6(6):e21610. http://dx.doi.org/10.1371/journal.pone.0021610

8. World Health Organization (WHO). Collecting, preserving and shipping specimens for the diagnosis of avian influenza $\mathrm{A}\left(\mathrm{H}_{5} \mathrm{~N}_{1}\right)$ virus infection 2006. Geneva: WHO. [Accessed 9 Sept 2011]. Available from: http://www.who.int/csr/resources/ publications/surveillance/CDS_EPR_ARO_2006_1.pdf

9. Waiboci LW, Lebo E, Williamson JM, Mwiti W, Kikwai GK, Njuguna $\mathrm{H}$, et al. Viral shedding in patients infected with pandemic influenza $A\left(\mathrm{H}_{1} \mathrm{~N}_{1}\right)$ virus in Kenya, 2009. PLoS One. 2011;6(6):e20320. http://dx.doi.org/10.1371/journal. pone.0020320

10. Shu B, Wu KH, Emery S, Villanueva J, Johnson R, Guthrie $E$, et al. Design and performance of the CDC real-time reverse transcriptase $P C R$ swine flu panel for detection of $2009 \mathrm{~A}\left(\mathrm{H}_{1} \mathrm{~N}_{1}\right)$ pandemic influenza virus. J Clin Microbiol. 2011;49(7):2614-9. http://dx.doi.org/10.1128/JCM.02636-10

11. Fiore AE, Shay DK, Broder K, Iskander JK, Uyeki TM, Mootrey $G$, et al. Prevention and control of influenza: recommendations of the Advisory Committee on Immunization Practices (ACIP), 2008. MMWR Recomm Rep. 2008;57(RR-7):1-60. http://dx.doi. org/rr5707a1

12. Wisconsin Veterinary Diagnostic Laboratory, Real time PCR values. Madison: University of Wisconsin; 2013. [Accessed 31 March 2014]. Available from: http://www.wvdl.wisc.edu/wpcontent/uploads/2013/01/WVDL.Info_.PCR_Ct_Values1.pdf

13. Waiboci LW, Arunga G, Mayieka LM, Kikwai G, Wakhule L, Shigoli MT, et al. Influenza surveillance in Kenya, 2008 - 2011: A low likelihood of successful subtyping and virus isolation for influenza positive specimens with high cycle threshold (CT) values. 3rd Annual African Network for Influenza Surveillance and Epidemiology (ANISE); 2012 Feb 1-3,; Nairobi, Kenya.

14. Baxter BD, Couch RB, Greenberg SB, Kasel JA. Maintenance of viability and comparison of identification methods for influenza and other respiratory viruses of humans. J Clin Microbiol. 1977;6(1):19-22.

15. Munster VJ, Baas C, Lexmond P, Bestebroer TM, Guldemeester J, Beyer WE, et al. Practical considerations for high-throughput influenza A virus surveillance studies of wild birds by use of molecular diagnostic tests. J Clin Microbiol. 2009;47(3):66673. http://dx.doi.org/10.1128/JCM.01625-08

16. Krafft AE, Russell KL, Hawksworth AW, McCall S, Irvine M Daum LT, et al. Evaluation of PCR testing of ethanol-fixed nasal swab specimens as an augmented surveillance strategy for influenza virus and adenovirus identification. J Clin Microbiol. 2005;43(4):1768-75. http://dx.doi.org/10.1128/ JCM.43.4.1768-1775.2005

17. Druce J, Garcia K, Tran T, Papadakis G, Birch C. Evaluation of swabs, transport media, and specimen transport conditions for optimal detection of viruses by PCR. J Clin Microbiol. 2012;50(3):1064-5. http://dx.doi.org/10.1128/JCM.06551-11 